\title{
Influência de Variáveis de Sinterização na Microestrutura de Peças de PTFE Moldadas por Prensagem Isostática
}

\author{
Patrícia Giunchetti Strabelli, Vinicius Fiocco Sciuti, Fernanda Silveira Montilha \\ Programa de Pós-Graduação em Ciência e Engenharia de Materiais, UFSCar \\ Leonardo Bresciani Canto, Rodrigo Bresciani Canto
Departamento de Engenharia de Materiais, UFSCar
}

Resumo: O politetrafluoretileno (PTFE) é utilizado em extensa gama de aplicações críticas devido à sua excelente resistência química e térmica, baixa energia superficial e propriedades tribológicas. Devido à sua elevada viscosidade no estado fundido, o PTFE não pode ser transformado pelos métodos tradicionais de extrusão e injeção, sendo o principal método de transformação deste polímero a prensagem sob temperatura ambiente, seguida de sinterização a temperaturas acima do ponto de fusão. $\mathrm{O}$ tempo de sinterização é definido de acordo com as dimensões da peça fabricada, podendo variar de poucas horas até vários dias para peças de grande volume. Avaliações sobre a influência dos extremos de tempo e temperatura de sinterização, atualmente adotados na indústria, sobre a microestrutura cristalina do PTFE, são escassas na literatura científica, sendo o principal objetivo deste estudo. Placas em PTFE foram sinterizadas variando-se a temperatura entre $360^{\circ} \mathrm{C} \mathrm{e} 390^{\circ} \mathrm{C}$ e o tempo entre 10 e $10.000 \mathrm{~min}$. Calorimetria exploratória diferencial (DSC), medidas de perda de massa e de densidade e microscopia eletrônica de varredura (MEV) foram utilizadas. Os resultados das medidas de perda de massa indicaram que a degradação do PTFE aumenta com o tempo e temperatura de sinterização. Análises das entalpias de fusão e medidas de densidade apontam indiretamente a redução na massa molar e aumento no grau de cristalinidade com o aumento do tempo e temperatura de sinterização. As análises em MEV possibilitaram a observação direta da microestrutura cristalina, indicando uma tendência de aumento da largura das lamelas com o tempo e temperatura de sinterização. Os resultados obtidos podem auxiliar no controle da microestrutura do PTFE durante o processamento, o que é bastante útil para a fabricação de peças em PTFE com desempenho otimizado.

Palavras-chave: PTFE, sinterização, degradação térmica, cristalinidade.

\section{Effects of Sintering Variables on the Microstructure of Isostatically Pressed PTFE Parts}

\begin{abstract}
Polytetrafluorethylene (PTFE) is used in a wide range of critical applications because of its outstanding chemical and thermal resistance, low surface energy and special tribological properties. Because of its high melt viscosity, PTFE cannot be processed by traditional extrusion and injection methods. Cold pressing followed by sintering above its melting temperature is the main processing technique. The sintering time is defined according to the dimensions of the manufactured part and may last from a few hours to several days for large parts. Studies of the influence of sintering time and temperature on the PTFE crystalline microstructure are scarce in the scientific literature, being the main objective of this study. PTFE plates were isostatically pressed and sintered at temperatures from $360^{\circ} \mathrm{C}$ and $390{ }^{\circ} \mathrm{C}$ for periods of times between 10 e 10,000 min. Differential Scanning Calorimetry (DSC), measurements of mass loss and density and Scanning Electron Microscopy (SEM) were performed. The results of mass loss indicated that degradation increases with sintering time and temperature. The analysis of the enthalpies of fusion and density measurements indicates indirectly the reduction in molecular weight and increase in the crystallinity degree with increasing sintering temperature and time. The SEM analysis allowed for direct observation of crystalline microstructure, indicating a trend of increasing width for the crystalline lamellae with sintering time and temperature. These data allow one to devise ways to tune the PTFE microstructure during processing, which is useful for the manufacturing of PTFE parts with optimized performance.
\end{abstract}

Keywords: PTFE, sintering, thermal degradation, crystallinity.

\section{Introdução}

O politetrafluoretileno (PTFE), conhecido principalmente pela marca comercial Teflon ${ }^{\circledR}$, foi desenvolvido no final da segunda guerra mundial no contexto do projeto Manhattan pela DuPont, sendo hoje fabricado por diversas empresas e utilizado em aplicações como revestimentos antiaderentes, isolamento elétrico de fios e cabos e peças técnicas, como anéis de vedação em sistemas automotivos e sedes de válvulas. É um polímero

com características diferenciadas oriundas de sua estrutura molecular composta por átomos de flúor ligados a uma extensa cadeia carbônica.

A elevada energia envolvida em cada ligação carbonoflúor, $116 \mathrm{kcal} \mathrm{mol}^{-1}$ comparada a 99,5 $\mathrm{kcal} \mathrm{mol}^{-1}$ para a ligação carbono-hidrogênio e $83 \mathrm{kcal} \mathrm{mol}^{-1}$ para a ligação carbono-carbono, confere ao PTFE estabilidade química e térmica excepcionais. O par de elétrons envolvidos na 
ligação carbono-flúor fica deslocalizado para o átomo de flúor devido à maior eletronegatividade deste elemento, todavia, como a cadeia polimérica do PTFE é simétrica e sem quaisquer ramificações, há um equilíbrio de cargas em cada mero que torna a molécula apolar e faz com que as interações intermoleculares sejam somente por forças dispersivas fracas. Tais características, associadas à "blindagem estérica" da cadeia carbônica pelos átomos de flúor, conferem ao PTFE anti-aderência, baixo coeficiente de atrito e alta capacidade de isolamento elétrico e térmico em relação aos demais polímeros ${ }^{[1]}$. Devido à fraca interação intermolecular por forças de dispersão no PTFE, para que o mesmo apresente propriedades mecânicas adequadas, é necessária uma elevada massa molar (MM), entre $10^{6}$ e $10^{7} \mathrm{~g}$ $\mathrm{mol}^{-1}$. Essas características conferem ao PTFE viscosidade de $10^{10}$ a $10^{12} \mathrm{cP}$ a $380{ }^{\circ} \mathrm{C}$, um milhão de vezes superior ao que se recomenda para métodos de transformação no estado fundido ${ }^{[1]}$. Isso inviabiliza processos convencionais de extrusão e injeção no estado fundido, de forma que um dos principais métodos de transformação deste material é a prensagem a frio (tipicamente a $35 \mathrm{MPa}$ ), seguida de sinterização sob pressão atmosférica ${ }^{[1]}$.

Após a polimerização, o PTFE apresenta grau de cristalinidade entre 89 e $98 \% \%^{[2,3]}$ e ponto de fusão de aproximadamente $342{ }^{\circ} \mathrm{C}$. Após passar por uma primeira fusão, durante a sinterização, o ponto de fusão do PTFE cai para valores ao redor de $327{ }^{\circ} \mathrm{C}^{[4,5]}$. De acordo com Kostromina et al. ${ }^{[4]}$, tal alteração estaria relacionada a uma redução na perfeição da fase cristalina após a primeira fusão. O grau de cristalinidade também é diminuído após a primeira fusão, ficando entre $30 \%$ e $70 \%$. Isso ocorre devido à elevada MM do PTFE, que leva à alta densidade de entrelaçamento das cadeias no estado fundido, dificultando a cristalização após a primeira fusão.

A estrutura cristalina do PTFE é do tipo lamelas empilhadas, formada por extensas cadeias dobradas intercaladas com fase amorfa. $\mathrm{O}$ comprimento das pilhas de lamelas e a largura das lamelas do PTFE são de aproximadamente $100 \mu \mathrm{m}$ e $1 \mu \mathrm{m}^{[1,6]}$. A morfologia da fase cristalina do PTFE tem relação com sua MM, dado que moléculas mais curtas possuem maior mobilidade e podem assumir a conformação ordenada, a qual compõe a fase cristalina, com maior facilidade ${ }^{[7,8]}$.

O processo de prensagem a frio consiste em compactar mecanicamente o PTFE sob forma de pó em temperatura ambiente $\left(\approx 25^{\circ} \mathrm{C}\right)$ com prensa uniaxial ou isostática, aplicando-se pressões, sob taxas controladas, com a finalidade de aproximar os grãos do polímero para permitir que a difusão entre os mesmos na sinterização seja suficiente para formar um sólido com baixa porosidade ${ }^{[5]}(<1 \%)$. Após a prensagem, a peça moldada deverá descansar sob pressão e temperatura ambiente para que o ar, ainda preso entre os grãos do PTFE, seja liberado. Passado o tempo de descanso, a peça "verde" será sinterizada sob pressão atmosférica e temperaturas recomendadas pelos fabricantes da matéria prima entre $355^{\circ} \mathrm{C}$ e $375^{\circ} \mathrm{C}$, para compostos de PTFE com cargas, e ao redor de $375^{\circ} \mathrm{C}$, para o PTFE puro ${ }^{[3,9]}$. A pressão e taxa de prensagem, o tempo de descanso, o tempo e a temperatura de sinterização irão variar dependendo do tipo de PTFE utilizado, assim como das dimensões da peça a ser fabricada. Quanto maior a temperatura e tempo de sinterização, menor será o nível de porosidade final ${ }^{[5]}$, porém, será maior a probabilidade de cisão das macromoléculas devido à degradação térmica ${ }^{[10]}$. Durante a sinterização define-se também a microestrutura e o grau de cristalinidade do polímero. Porosidade, degradação térmica e cristalinidade são características importantes para a definição das propriedades mecânicas do $\mathrm{PTFE}^{[1]} \mathrm{e}$, assim sendo, torna-se valioso que os parâmetros utilizados pela indústria na sinterização de peças de PTFE com diversas dimensões proporcionem as propriedades adequadas para o material e seu bom desempenho em uso.

Embora o PTFE seja um polímero de engenharia de grande importância tecnológica no mercado mundial e nacional, há poucos trabalhos no Brasil, com exceção para o estudo sobre processamento do grupo do Professor A. S. Gomes $^{[9]}$, os trabalhos sobre modificação química do grupo do Professor A. B. Lugão ${ }^{[12-15]}$ e os nossos estudos que abordam os processos de prensagem a frio e sinterização do PTFE ${ }^{[3,5,16-18]}$ e de laminados compósitos de PTFE com tecidos de fibra de vidro ${ }^{[19,20]}$.

O presente trabalho tem como objetivo investigar a influência de variáveis envolvidas na etapa de sinterização, a saber, tempo e temperatura máxima de sinterização, na microestrutura cristalina do PTFE. Os resultados deste trabalho irão proporcionar embasamento científico para a determinação dos limites para as variáveis atualmente adotadas pela indústria para sinterizar peças em PTFE, verificando que sejam adequadas para proporcionar propriedades mecânicas satisfatórias no polímero, que, dependendo das dimensões da peça, pode ser exposto à temperatura máxima de sinterização por tempos tão curtos quanto $10 \mathrm{~min}$, ou longos quanto $10.000 \mathrm{~min}$, sob os recomendados $375^{\circ} \mathrm{C}$, incluindo variações de aproximadamente $15^{\circ} \mathrm{C}$ para mais ou para menos, devido às heterogeneidades comumente encontradas nos fornos industriais.

\section{Experimental}

\section{Material}

O material em estudo é o PTFE puro - Teflon ${ }^{\circledR} 807-\mathrm{N}$ da DuPont, recebido em forma de grânulos (ou pellets), com granulometria média aproximada de $600 \mu \mathrm{m}$ e uma porosidade (razão entre o volume de vazios e o volume do sólido) de $\approx 60 \%$. Foi polimerizado em suspensão aquosa seguida de um processo de corte e peletização para que os grãos tomem a forma esferoidal, que facilita o preenchimento de moldes na etapa de prensagem a frio.

\section{Preparação das amostras de PTFE}

A preparação das amostras de PTFE foi realizada pelas seguintes etapas:

1. Prensagem isostática de placas com dimensões, após prensagem, de $\approx 7.20 .80 \mathrm{~mm}^{3}$;

2. Sinterização individual das placas segundo 12 condições de tratamento térmicos distintos, realizadas em forno com controle computadorizado de temperatura, 
circulação forçada de ar que garante variações no controle $<2{ }^{\circ} \mathrm{C}$;

3. Corte das placas com lâminas de aço para a obtenção das amostras para as análises térmicas e de microscopia.

No processo de prensagem isostática o material é depositado em um molde elastomérico flexível, posteriormente selado e inserido em um vaso de pressão onde é submetido a um carregamento devido à pressurização de um fluido até uma determinada pressão. As placas foram prensadas em múltiplos passos para a eliminação gradativa do ar presente no molde seguindo um procedimento descrito por Gamboni et al. ${ }^{[17]}$ e esquematizado na Figura 1. O material foi processado com uma pressão inicial de $2 \mathrm{MPa}$ para obtenção da pré-forma, uma prensagem intermediária com pressão de $10 \mathrm{MPa}$ e a prensagem final com uma pressão de $35 \mathrm{MPa}$, que é o valor recomendado pelo fabricante da matéria-prima. Em todas as etapas as pressões foram mantidas por 1 min e o tempo de descanso para a liberação do ar residual entre as prensagens foi de no mínimo $6 \mathrm{~h}$.

Na Figura 1 também é apresentada a curva de tratamento térmico padrão que foi aplicada no processo de sinterização do PTFE. Esta curva foi definida em função das dimensões das peças utilizadas para garantir que o gradiente de temperaturas no interior das placas seja insignificante, e assim, evitar o trincamento da peça durante este processo ${ }^{[18]}$. As variações no tratamento térmico foram na temperatura máxima de sinterização, $T_{S}$, e no tempo de sinterização, $t_{s}$, ou seja, o tempo do patamar sob a temperatura máxima. Foram adotadas 12 condições principais de sinterização neste estudo, resumidas na Tabela 1.

Para as três temperaturas $T_{S}\left(360,375\right.$ e $\left.390{ }^{\circ} \mathrm{C}\right)$ foram aplicados quatro diferentes tempos $t_{S}(10,120$, 1.000 e $10.000 \mathrm{~min})$. Uma destas condições, $T_{S}=375^{\circ} \mathrm{C}$ e $t_{S}=120 \mathrm{~min}$, é a recomendada pelo fabricante da matéria-prima para as dimensões das placas estudadas. As temperaturas de sinterização de $360{ }^{\circ} \mathrm{C}$ e $390{ }^{\circ} \mathrm{C}$ foram estipuladas considerando que variações de $\pm 15^{\circ} \mathrm{C}$ são possíveis de serem encontradas na indústria devido ao tamanho elevado e a consequente limitação na homogeneidade dos fornos industriais. $\mathrm{O}$ menor valor de $t_{S}(10 \mathrm{~min})$ foi escolhido para representar possíveis regiões internas de peças volumosas que possam não estar sujeitas ao tempo total do tratamento térmico devido à baixa condutividade térmica do $\mathrm{PTFE}^{[18]}$. Por outro lado, o maior valor de $t_{S}(10.000 \mathrm{~min})$ foi escolhido para representar possíveis regiões externas de peças volumosas que necessitem de longos tempos de sinterização para que seu interior permaneça na temperatura de sinterização pelo tempo mínimo estipulado no planejamento do processo.

\section{Análise de perda de massa}

A perda de massa do PTFE, por decomposição térmica, foi estudada por meio de medição da massa das placas submetidas aos diferentes ciclos de sinterização descritos na Tabela 1. Um mínimo de três placas foi fabricado para cada uma das 12 condições e a atmosfera utilizada na sinterização de todas as amostras foi a ambiente.

\section{Medidas de densidade}

Medidas da densidade foram realizadas para correlacioná-las com as condições de sinterização e os valores também foram utilizados para o cálculo indireto do grau de cristalinidade.

Foi utilizado o método de submersão em água e cálculo do empuxo (princípio de Arquimedes). Para minimizar os erros na comparação das condições de processamento, as amostras foram usinadas a partir da região central de cada placa, resultando em paralelepípedos com dimensões

Tabela 1. Principais condições de sinterização estudadas.

\begin{tabular}{ccc}
\hline Condição & $\begin{array}{c}\text { Temperatura, } \boldsymbol{T}_{s} \\
{ }^{\mathbf{C}}\end{array}$ & $\begin{array}{c}\text { Tempo, } \boldsymbol{t}_{s} \\
\mathbf{\text { min }}\end{array}$ \\
\hline 1 & 360 & 10 \\
2 & 360 & 120 \\
3 & 360 & 1.000 \\
4 & 360 & 10.000 \\
5 & 375 & 10 \\
6 & 375 & 120 \\
7 & 375 & 1.000 \\
8 & 375 & 10.000 \\
9 & 390 & 10 \\
10 & 390 & 120 \\
11 & 390 & 1.000 \\
12 & 390 & 10.000 \\
\hline
\end{tabular}

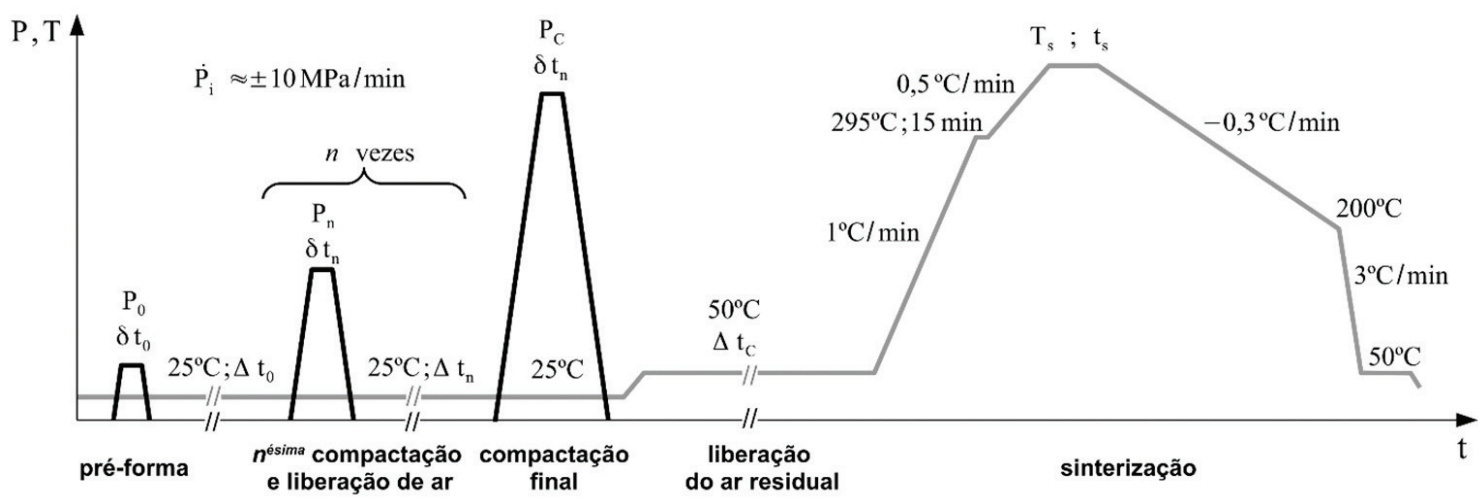

Figura 1. Representação das rampas e patamares de pressão isostática empregados na fabricação das placas e curva típica de tratamento térmico para os processos de sinterização adotados. 
de $5 \cdot 5 \cdot 10 \mathrm{~mm}^{3}$. Foram avaliadas três amostras para cada condição de sinterização.

\section{Calorimetria exploratória diferencial - DSC}

Os experimentos de DSC foram realizados em um equipamento da TA Instruments, modelo Q2000. Foram realizados ensaios com 2 ciclos de aquecimento e resfriamento no material sinterizado com o objetivo de avaliar a entalpia de fusão e cristalização nas placas sinterizadas sob as 12 condições principais de processamento. $\mathrm{O}$ ciclo aplicado foi: temperatura mínima (ou do patamar inferior) de $180{ }^{\circ} \mathrm{C}$ com patamar de $5 \mathrm{~min}$; taxa de aquecimento/ resfriamento de $10{ }^{\circ} \mathrm{C} \mathrm{min}^{-1}$; temperatura máxima (ou do patamar superior) de $380{ }^{\circ} \mathrm{C}$ com patamar de $5 \mathrm{~min}$.

\section{Microscopia Eletrônica de Varredura - MEV}

A técnica de microscopia eletrônica de varredura (MEV) foi utilizada para investigar e comparar a microestrutura da fase cristalina das placas de PTFE submetidas às diferentes condições de sinterização. Foram extraídas, das regiões internas das placas, amostras na forma de paralelepípedo com medidas de aproximadamente 3.3.20 $\mathrm{mm}^{3}$. Essas amostras foram criofraturadas e uma fatia de aproximadamente $3 \mathrm{~mm}$ de espessura foi cortada e colada no porta amostras. As amostras foram fixadas e aterradas com "tinta prata" em suporte de alumínio e após a secagem por $\approx 3 \mathrm{~h}$ foram metalizadas com ouro no equipamento marca Balzers, modelo Sputter Coater SCD 004. O equipamento utilizado para a microscopia foi um microscópio da $F E I$ Company, modelo Magellan $400 L$, tipo FEG, operando com tensões aceleradoras do feixe de elétrons entre 2 e $5 \mathrm{kV}$. As imagens obtidas em MEV foram analisadas com o programa ImageJ para a medição da largura das lamelas do PTFE em função das condições de sinterização.

\section{Resultados e Discussões}

\section{Perda de massa}

Visando verificar a influência da temperatura e do tempo de sinterização na degradação térmica do PTFE, as placas sinterizadas nas 12 condições (Tabela 1) foram pesadas antes e depois da sinterização. Os resultados dos valores médios das perdas de massa em função do tempo sob a temperatura máxima são apresentados na Figura 2. Pelo menos três amostras foram medidas para cada condição e o desvio padrão máximo obtido foi para a condição mais crítica $\left(10.000\right.$ min sob $\left.390{ }^{\circ} \mathrm{C}\right)$, não ultrapassando $0,1 \% \mathrm{e}$, para as outras condições, não ultrapassando $0,02 \%$, o que viabilizou a análise do gráfico (as barras de desvio padrão não foram mostradas para facilitar a leitura do gráfico).

Foi possível identificar uma perda de massa, mesmo em temperaturas abaixo de $400{ }^{\circ} \mathrm{C}$, o que não é viável medir com uma simples análise termogravimétrica (TG) padrão. Nestas análises, degradações por perda de massa no PTFE somente são observadas acima de $450{ }^{\circ} \mathrm{C}^{[21]}$, uma vez que a amostra é submetida a uma determinada temperatura durante um curto espaço de tempo. Dessa forma, o procedimento adotado, apesar de simples, proporcionou a obtenção de resultados importantes.

Os valores de perda de massa medidos ficaram abaixo de $1 \%$ mesmo para a condição mais crítica (10.000 min sob $390{ }^{\circ} \mathrm{C}$ ). Apesar de os valores serem relativamente baixos, considerando o grande tempo de exposição sob altas temperaturas, foi possível verificar uma relação direta entre tempo e temperatura de sinterização e a decomposição térmica do PTFE. Para tempos inferiores a $1.000 \mathrm{~min}$, a perda de massa foi próxima a zero para as temperaturas de $360{ }^{\circ} \mathrm{C}$ e $375^{\circ} \mathrm{C}$ e próxima a $0,1 \%$ para a temperatura de $390{ }^{\circ} \mathrm{C}$. Para o tempo de $10.000 \mathrm{~min}$, foi possível observar uma perda de massa considerável em todas as temperaturas. Sob $360^{\circ} \mathrm{Ce} 375^{\circ} \mathrm{C}$, a perda de massa foi, respectivamente, de $0,2 \%$ e $0,4 \%$, alcançando $0,95 \%$ para $390{ }^{\circ} \mathrm{C}$.

Todavia, antes que a cadeia do PTFE seja reduzida ao nível de compostos voláteis que levam à perda de massa, há redução na massa molar (MM) por cisão das cadeias com formação de segmentos mais curtos, não voláteis, que podem influenciar as propriedades do PTFE.

\section{Medidas de densidade}

Medidas de densidade foram efetuadas para investigar o efeito das 12 condições de sinterização nesta propriedade. Os resultados obtidos para cada condição e desvio padrão são apresentados na Figura 3.

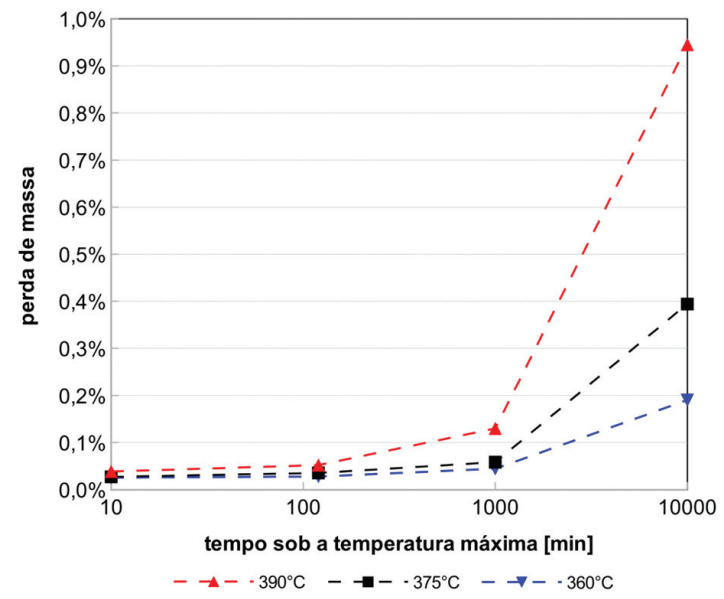

Figura 2. Perda de massa para as placas de PTFE sinterizadas por $10,120,1.000$ e $10.000 \mathrm{~min}$, sob $360^{\circ} \mathrm{C}, 375^{\circ} \mathrm{C}$ e $390{ }^{\circ} \mathrm{C}$.

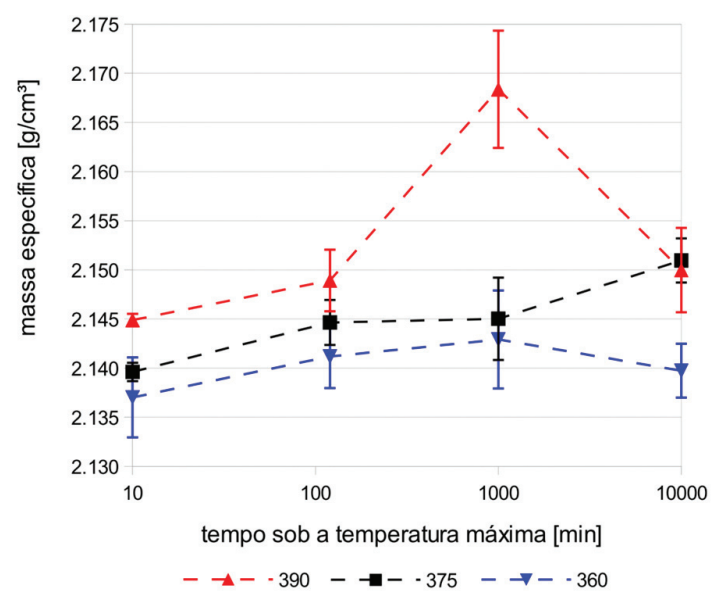

Figura 3. Medidas de densidade para amostras de PTFE obtidas das placas sinterizadas por 10, 120, 1.000 e $10.000 \mathrm{~min}$, sob 360 ${ }^{\circ} \mathrm{C}, 375{ }^{\circ} \mathrm{C}$ e $390{ }^{\circ} \mathrm{C}$. 
O aumento da densidade está associado principalmente ao aumento do grau de cristalinidade quando o material é exposto de forma prolongada às altas temperaturas - como será discutido adiante - uma vez que a porosidade residual do processo de prensagem a frio utilizado é muito baixa $(\approx 1,5 \%)^{[5]}$. Considerando as densidades da fase amorfa $\left(\rho_{A}=\right.$ $\left.2,00 \mathrm{~g} \mathrm{~cm}^{-3}\right)$ e da fase cristalina $\left(\rho_{C}=2,30 \mathrm{~g} \mathrm{~cm}^{-3}\right)^{[22]}$ e ainda que a diferença dos extremos dos valores de densidades medidos - de $2,138 \mathrm{~g} \mathrm{~cm}^{-3}\left(360^{\circ} \mathrm{C} ; 10 \mathrm{~min}\right)$ e $2,168 \mathrm{~g} \mathrm{~cm}^{-3}$ $\left(390^{\circ} \mathrm{C} ; 1.000 \mathrm{~min}\right)$ - seja atribuída somente à alteração no grau de cristalinidade, estas amostras apresentariam graus de cristalinidade de $45,7 \%$ e $56,1 \%$, respectivamente, ou seja, haveria alteração no grau de cristalinidade de aproximadamente $10 \%$ em função das condições de sinterização adotadas, o que pode ser relevante para as propriedades do material.

\section{Calorimetria diferencial exploratória (DSC)}

Ensaios de DSC com dois ciclos de aquecimento e resfriamento foram realizados nas placas de PTFE sinterizadas segundo as 12 condições (Tabela 1). Para possibilitar uma comparação precisa no cálculo da área sob os picos de transformação de fase, foram tomadas linhas de base sempre com as mesmas temperaturas em seus extremos, sendo de 200 a $375^{\circ} \mathrm{C}$ para os picos de fusão e de 340 a $200{ }^{\circ} \mathrm{C}$ para os picos de cristalização.

Nestes ensaios, o segundo aquecimento foi executado para obter-se o valor da entalpia de fusão livre do histórico térmico do processo de sinterização da placa. Dessa forma, esta segunda fusão em DSC permite evidenciar somente o efeito da variação na MM do PTFE nos valores de entalpia medidos. Por outro lado, os dois resfriamentos servem de base para verificar a repetibilidade alcançada nas medidas de entalpia de cristalização em uma mesma amostra.

Os resultados das entalpias dos picos de fusão durante os primeiros aquecimentos, em função do tempo de permanência sob as temperaturas máximas de 360, 375 e $390{ }^{\circ} \mathrm{C}$, são apresentados na Figura 4. Observa-se um comportamento análogo ao obtido pelas medidas de densidade apresentadas na Figura 3. É possível verificar a influência de ambas as variáveis, temperatura e tempo, sobre o grau de cristalinidade das amostras, notando-se uma tendência de aumento das entalpias e, portanto, do grau de cristalinidade com o aumento da temperatura e do tempo de sinterização. Novamente, algumas exceções são observadas principalmente para a condição de $10.000 \mathrm{~min}$ sob $390{ }^{\circ} \mathrm{C}$, em que a entalpia cai para o mesmo nível da condição de 10.000 min sob $375^{\circ} \mathrm{C}$.

Os resultados das entalpias nos picos de fusão durante os segundos aquecimentos, em função do tempo de permanência sob as temperaturas máximas de 360 , 375 e $390^{\circ} \mathrm{C}$, são apresentados na Figura 5. Observa-se que, qualitativamente, os resultados são análogos aos do primeiro aquecimento. Porém, em termos quantitativos, as diferenças nas entalpias são menores. Isto é devido à maior taxa de resfriamento aplicada nos ensaios de DSC $\left(10^{\circ} \mathrm{C} \mathrm{min}^{-1}\right)$ quando comparada à taxa de resfriamento menor utilizada na sinterização das placas $\left(0,3{ }^{\circ} \mathrm{C} \mathrm{min}^{-1}\right)$, ou seja, esta taxa de resfriamento maior no ensaio de DSC resulta em um grau de cristalinidade menor.
Os resultados das entalpias nos picos de cristalização durante os primeiros e segundos resfriamentos em função do tempo de permanência sob as temperaturas máximas ficaram muito próximos dos resultados apresentados na Figura 5 para o segundo aquecimento, o que revela a repetibilidade do equipamento em ciclos de uma mesma amostra sob as mesmas taxas de aquecimento ou resfriamento.

As temperaturas dos picos de fusão variaram cerca de 1 ${ }^{\circ} \mathrm{C}$ em função das condições de sinterização, sendo que as da primeira fusão variaram entre 329,2 e $330,3{ }^{\circ} \mathrm{C}$, enquanto que as da segunda fusão variaram entre 328,2 e $329,2{ }^{\circ} \mathrm{C}$, ambas com comportamento análogo aos das respectivas entalpias. As temperaturas dos picos de cristalização nos primeiros e segundos resfriamentos variaram cerca de $3{ }^{\circ} \mathrm{C}$, entre 313,2 e $316,2{ }^{\circ} \mathrm{C}$, apresentando tendência de diminuição com o aumento do tempo de sinterização.

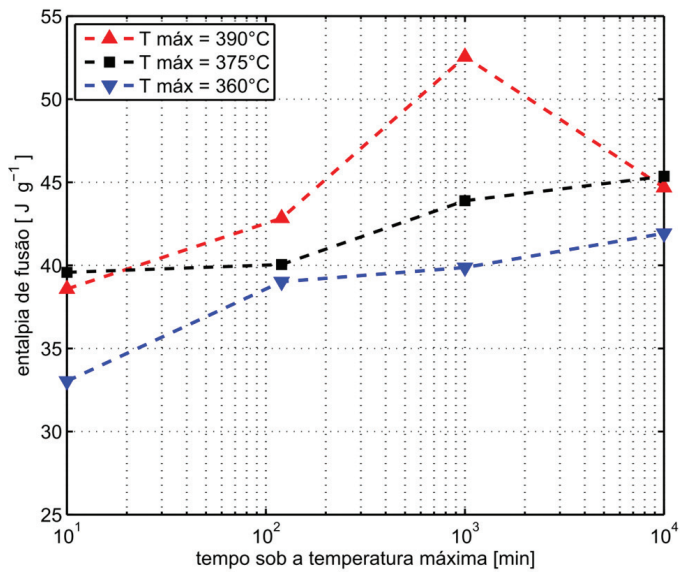

Figura 4. Entalpias de fusão do primeiro aquecimento em ensaio de DSC com dois ciclos de aquecimento e resfriamento, em amostras de PTFE sinterizadas segundo as 12 condições principais por 10 , $120,1.000$ e 10.000 min sob as temperaturas máximas de 360 , 375 e $390{ }^{\circ} \mathrm{C}$

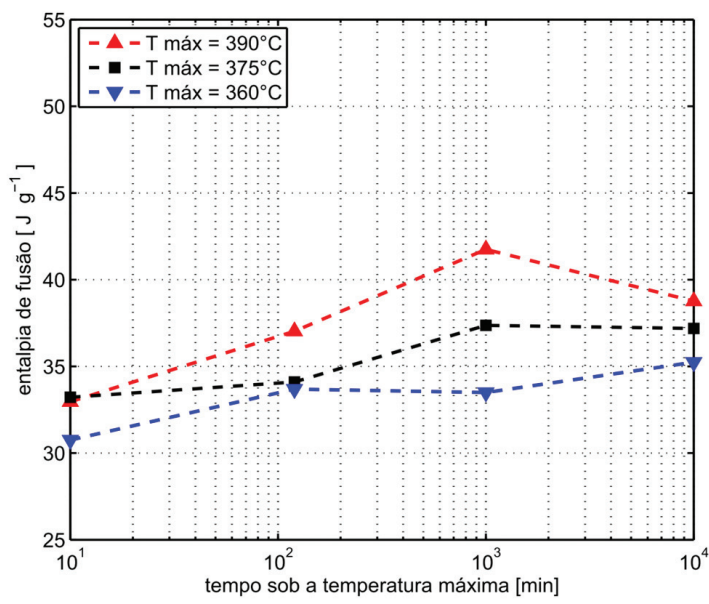

Figura 5. Entalpias de fusão do segundo aquecimento em ensaio de DSC com dois ciclos de aquecimento e resfriamento, em amostras de PTFE sinterizadas segundo as 12 condições principais por 10 , $120,1.000$ e 10.000 min sob as temperaturas máximas de 360 , 375 e $390^{\circ} \mathrm{C}$. 
A análise global do primeiro (Figura 4) e segundo (Figura 5) aquecimentos mostra que as variáveis de sinterização estudadas - tempo e temperatura - influenciam significativamente na cristalização do PTFE, notando-se uma tendência de aumento das entalpias e, portanto, do grau de cristalinidade com o aumento da temperatura e com o aumento do tempo de sinterização, o que corrobora com os resultados discutidos anteriormente nas medições de densidades. As exceções a esta tendência, observadas principalmente para a condição de sinterização sob $390{ }^{\circ} \mathrm{C}$ por $10.000 \mathrm{~min}$, podem estar associadas ao fenômeno de nucleação do processo de cristalização induzido pela presença de resquícios da fase cristalina original oriunda da polimerização, que diminuem gradualmente ao longo da sinterização.

Se a diferença dos extremos dos valores de entalpias de fusão do primeiro aquecimento (Figura 4), de 33, $0 \mathrm{~J} \mathrm{~g}^{-1}$ $\left(360{ }^{\circ} \mathrm{C} ; 10 \mathrm{~min}\right)$ e $52,6 \mathrm{~J} \mathrm{~g}^{-1}\left(390{ }^{\circ} \mathrm{C} ; 1.000 \mathrm{~min}\right)$, for atribuída somente a uma alteração no grau de cristalinidade, considerando uma entalpia de referência de $80 \mathrm{~J} \mathrm{~g}^{-1[11]}$, estas amostras apresentariam graus de cristalinidade de 41,3\% e $65,6 \%$, respectivamente, ou seja, haveria uma alteração no grau de cristalinidade de aproximadamente $24 \%$, o que pode ser considerável em termos de propriedades do material final. Tal diferença foi muito superior a encontrada para o cálculo do grau de cristalinidade pelas medidas de densidade, que foi de aproximadamente 10\%. Esta discrepância é comumente encontrada na literatura quando se avalia o grau de cristalinidade por diferentes técnicas ${ }^{[11]}$.

É sabido que a entalpia de fusão e então o grau de cristalinidade do PTFE aumentam com a degradação por cisão das cadeias, a qual aumenta a mobilidade e a facilidade de cristalização no resfriamento após a etapa de sinterização. Assim, amostras sinterizadas em condições mais severas apresentaram maior grau de cristalinidade. Exceção se faz a amostra sinterizada sob $390{ }^{\circ} \mathrm{C}$ por $10.000 \mathrm{~min}$ que foi influenciada por outro mecanismo discutido anteriormente.

\section{Microscopia Eletrônica de Varredura (MEV)}

A técnica de MEV foi empregada para observar a estrutura da fase cristalina do PTFE sinterizado segundo as 12 condições de processamento $(10,120,1.000 \mathrm{e}$ $10.000 \mathrm{~min}$, sob $360{ }^{\circ} \mathrm{C}, 375^{\circ} \mathrm{C}$ e $390{ }^{\circ} \mathrm{C}$ ). A análise

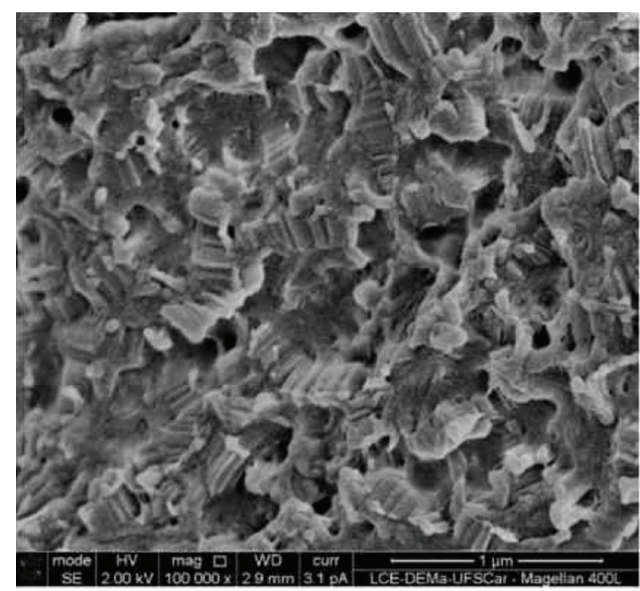

a) $390^{\circ} \mathrm{C}, 10 \mathrm{~min}$

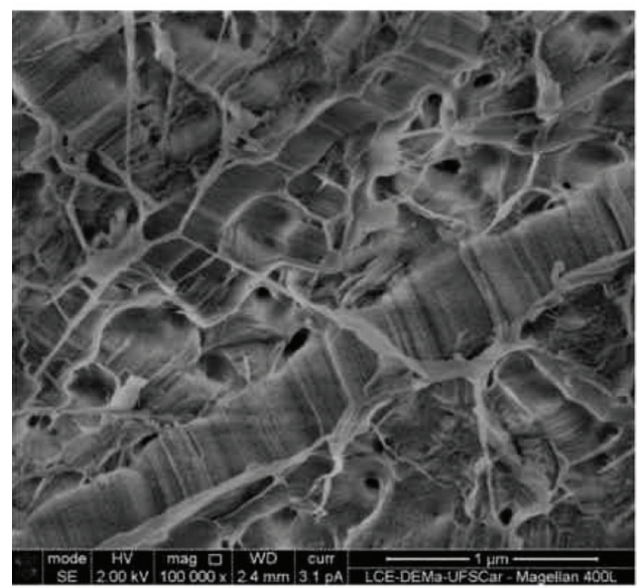

c) $390^{\circ} \mathrm{C}, 1.000 \mathrm{~min}$

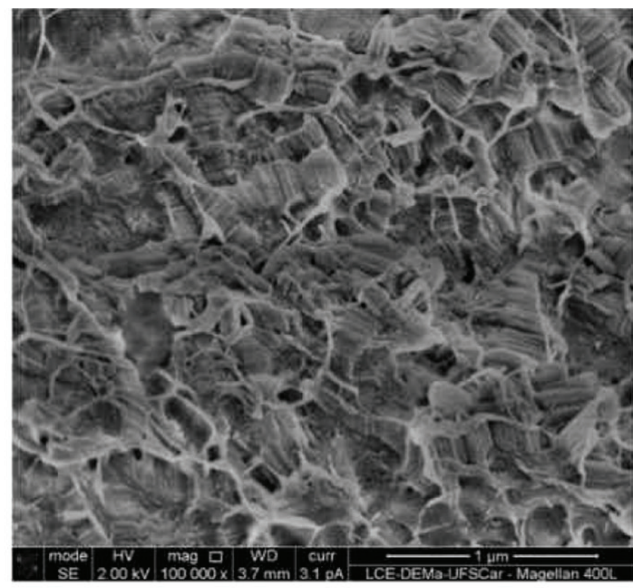

b) $390^{\circ} \mathrm{C}, 120 \mathrm{~min}$

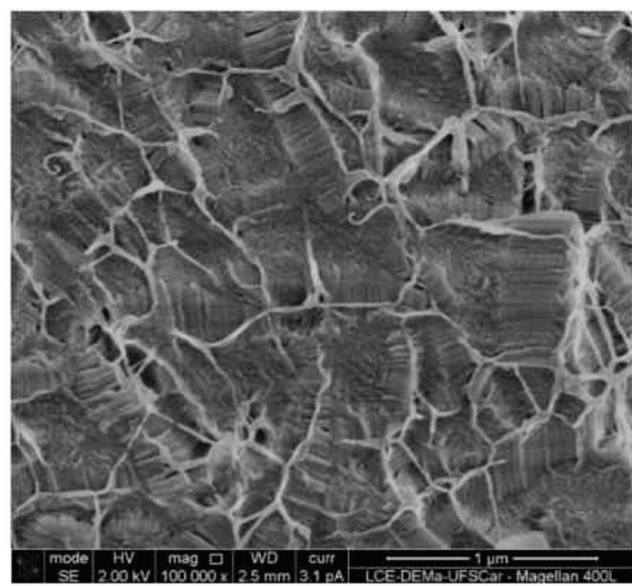

d) $390^{\circ} \mathrm{C}, 10.000 \mathrm{~min}$

Figura 6. Fotomicrografias de MEV das superfícies de amostras crio-fraturadas para as condições de sinterização: temperatura de $390{ }^{\circ} \mathrm{C}$ por (a)10 min, (b)120 min, (c)1.000 min e (d)10.000 min. 
visual das imagens possibilitou primeiramente observar a presença, em todas as imagens, de fibrilas de PTFE combinadas com regiões amorfas e cristalinas, porém, com diferenças significativas entre as estruturas cristalinas formadas sob cada tempo de patamar e, principalmente, sob cada temperatura de sinterização.

Para exemplificar as análises de MEV, na Figura 6 são apresentadas as fotomicrografias das superfícies criofraturadas de amostras de PTFE sinterizadas a $390{ }^{\circ} \mathrm{C}$ em quatro tempos distintos. Para esta condição de sinterização, a visualização das mesmas é bem clara, podendo-se observar diferenças nas dimensões das estruturas cristalinas em função do tempo de sinterização. Fica evidente que na condição de 1.000 min as estruturas cristalinas apresentam dimensões maiores.

Buscando realizar uma análise dimensional estatística das estruturas cristalinas observadas nas 12 condições de sinterização, utilizou-se do programa ImageJ para efetuar medições das larguras das lamelas, os resultados são apresentados na Figura 7.

A observação das imagens permite verificar um aumento na largura das lamelas para maiores temperaturas e tempos de sinterização, o que é confirmado pelo gráfico apresentado na Figura 7. Particularmente para a temperatura de sinterização de $390{ }^{\circ} \mathrm{C}$ e para o tempo de $1.000 \mathrm{~min}$, é possível observar nitidamente a presença de lamelas mais largas na superfície analisada, o que também é verificado no gráfico da Figura 7. Deve-se destacar que as análises de DSC e densidade apontaram um maior grau de cristalinidade para esta condição de sinterização, assim, as análises em MEV vieram a confirmar, seja por observação direta ou pela análise comparativa das medidas dos cristais, o maior grau de cristalinidade obtido para a condição de sinterização sob $390{ }^{\circ} \mathrm{C}$ por 1.000 min.

Os valores médios de largura das lamelas variaram entre 125 e $275 \mathrm{~nm}$, para as condições de sinterização de $360^{\circ} \mathrm{C}$ por $10 \mathrm{~min}$ e $390^{\circ} \mathrm{C}$ por $1.000 \mathrm{~min}$, respectivamente. Os valores de espessura e largura das lamelas encontrados nas análises de MEV são compatíveis com os valores reportados na literatura ${ }^{[1,7,23]}$.

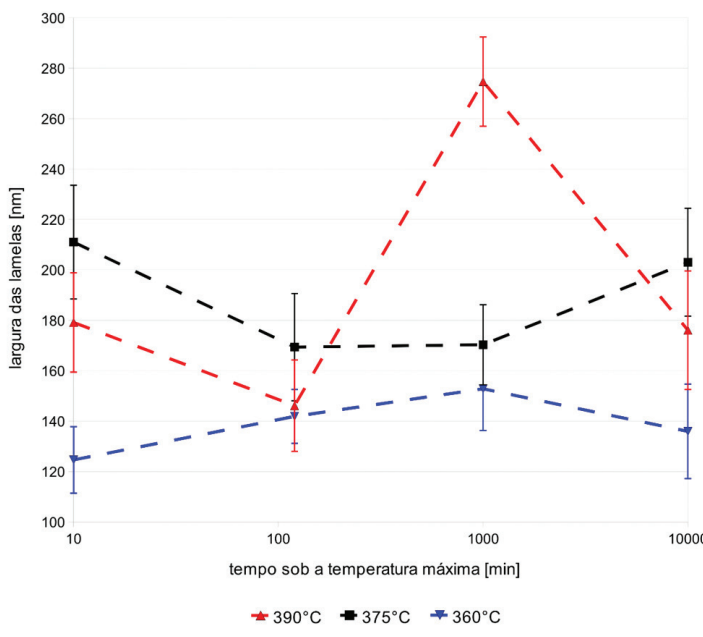

Figura 7. Largura das lamelas de PTFE sinterizado sob $360^{\circ} \mathrm{C}$ e $390{ }^{\circ} \mathrm{C}$ por $10,120,1.000$ e $10.000 \mathrm{~min}$.

\section{Conclusões}

Este estudo investigou os efeitos da temperatura e do tempo de sinterização sobre a degradação, o grau de cristalinidade e a microestrutura cristalina de peças de PTFE moldadas por prensagem isostática. Os parâmetros de sinterização estudados foram variados dentro da faixa utilizada na indústria, ou seja, em temperaturas entre 360 e $390{ }^{\circ} \mathrm{C}$ e tempos de sinterização entre 10 e $10.000 \mathrm{~min}$.

Os níveis de degradação do PTFE em função das condições de sinterização foram avaliados por medidas de perda de massa das peças de PTFE após a sinterização. Observou-se aumento da perda de massa com a temperatura e o tempo de sinterização, alcançando $0,02 \%$ para a condição mais branda $\left(360{ }^{\circ} \mathrm{C}\right.$ e $\left.10 \mathrm{~min}\right)$ e $1 \%$ para a condição mais severa de sinterização $\left(390^{\circ} \mathrm{C}\right.$ e $\left.10.000 \mathrm{~min}\right)$.

As densidades e os graus de cristalinidade (estimados a partir das entalpias de fusão em DSC) das peças moldadas em PTFE apresentaram tendência de aumento com a temperatura e o tempo sinterização. A amostra sinterizada a $390{ }^{\circ} \mathrm{C}$ durante $1.000 \mathrm{~min}$ apresentou densidade e entalpia de fusão $1,4 \%$ e $45,6 \%$ maiores, respectivamente, com relação à amostra sinterizada na condição mais branda $\left(360^{\circ} \mathrm{C}\right.$ e $\left.10 \mathrm{~min}\right)$. A densificação das amostras é o resultado do maior grau de coesão granular e do aumento do grau de cristalinidade pelo aumento da temperatura e do tempo de sinterização. O aumento da entalpia de fusão e então do grau de cristalinidade com a severidade da sinterização, por sua vez, sugere um maior nível de degradação por cisão das cadeias do PTFE, aumentando a mobilidade e a facilidade de cristalização no resfriamento após a etapa de sinterização.

A microestrutura cristalina das amostras de PTFE, constituída de lamelas empilhadas, foi analisada por MEV. $\mathrm{O}$ aumento da temperatura e do tempo de sinterização provocou variação aleatória e significativa na largura das lamelas, entre 120 e $280 \mathrm{~nm}$. A amostra sinterizada a $390{ }^{\circ} \mathrm{C}$ por 1.000 min apresentou o maior valor de largura das lamelas, corroborando os maiores valores de densidade e entalpia de fusão observados para esta condição de sinterização.

Do ponto de vista de aplicação tecnológica do PTFE, os resultados deste estudo permitem aos fabricantes de PTFE embasar suas recomendações em relação aos parâmetros de sinterização, sendo tais parâmetros a temperatura nominal de $375{ }^{\circ} \mathrm{C}$, tolerância de variações no forno de $15^{\circ} \mathrm{C}$ para cima ou para baixo e tempos de sinterização proporcionais à espessura da peça sinterizada, podendo chegar a $10.000 \mathrm{~min}$ para pré-formas com diâmetro superior a $1.000 \mathrm{~mm}$.

\section{Agradecimentos}

O presente trabalho foi realizado com apoio da FAPESP, Fundação de Amparo à Pesquisa do Estado de São Paulo (bolsas de pesquisa dos processos 2012/10317-9 e 2012/10319-1)

\section{Referências Bibliográficas}

1. Ebnesajjad, S. - "Non-Melt Processible Fluoroplastics", William Andrew Publishing, New York (2000). Fluoroplastics 1. 
2. Brown, E. N. \& Dattelbaum, D. M. - Polym., 46, p.3056 (2005). http://dx.doi.org/10.1016/j.polymer.2005.01.061.

3. Canto, R. B. - "Estudo teórico e experimental dos processos de compactação e sinterização do politetrafluoretileno (PTFE)", Tese de Doutorado, Universidade de São Paulo, Brasil/École Normale Supérieure de Cachan, França (2007).

4. Kostromina, S. V. - Polym. Sci., 32, p.388 (1990).

5. Canto, R. B.; Schmitt, N.; Carvalho, J. \& Billardon, R. Polym. Eng. Sci., 51, p.2220 (2011). http://dx.doi.org/10.1002/ pen.21994.

6. Speerscneider, C. J. \& Li, C. H. - J. Appl. Phys., 33, p.1871 (1962). http://dx.doi.org/10.1063/1.1728849.

7. Ting-Yung, H. \& Eiss Jr, N. S. - Wear, 84, p.203 (1983). http:// dx.doi.org/10.1016/0043-1648(83)90264-8.

8. Villani, V. - Thermochim. Acta., 162, p.189 (1990). http:// dx.doi.org/10.1016/0040-6031(90)80340-5.

9. Mantuano, T. R. A. \& Gomes, A. S. - Polímeros, p.15 (1994).

10. Yang, J.; Williams, R.; Peterson, K.; Geil, P. H.; Long, T.C. \& Xu, P. - Polym. J., 46, p.8723 (2005). http://dx.doi. org/10.1016/j.polymer.2005.01.108.

11. Rae, P. J. \& Dattelbaum, D. M. - Polym. J., 45, p.7615 (2004). http://dx.doi.org/10.1016/j.polymer.2004.08.064.

12. Moura, E. A. B.; Lugão, A. B.; Andrade e Silva, L. G. - Radiat. Phys. Chem., 52, p.229 (1998). http://dx.doi.org/10.1016/ S0969-806X(98)00145-5.

13. Ferreto, H. F. R.; Lima, L. F. C. P.; Parra, D. F.; Zaia, V. \& Lugão, A. B. - "Synthesis of polytetrafluoroethylene based olefinic copolymer by gamma radiation grafting. Nuclear Instruments and Methods in Physics Research Section B: Beam Interactions with Materials and Atoms", in: 7th International Symposium on Ionizing Radiation and Polymers, p.1, Antalya, Turkey, sept (2006).

14. Geraldes, A. N.; Zen, H. A.; Ribeiro, G.; Ferreira, H. P.; Souza, C. P.; Parra, D. F.; Santiago, E. I. \& Lugão, A. B. - Radiat. Phys. Chem., 79, p.246 (2010). http://dx.doi.org/10.1016/j. radphyschem.2009.08.029.

15. Napoleão Geraldes, A.; Augusto Zen, H.; Ribeiro, G.; Fernandes Parra, D. \& Benévolo Lugão, A. - Radiat. Phys. Chem., 84, p.205 (2013). http://dx.doi.org/10.1016/j. radphyschem.2012.06.016.
16. Melo, C. C.; Schmitt, N.; Carvalho, J.; Billardon, R. \& Canto, R. B. - "Identificação de parâmetros do processo de compactação do PTFE utilizando procedimento experimental associado à análise inversa pelo método dos elementos finitos", in: Anais do $20^{\circ}$ Congresso Brasileiro de Engenharia e Ciência dos Materiais, p.11638, Joinville - SC, nov (2012).

17. Gamboni, O. C.; Riul, C.; Schmitt, N.; Billardon, R.; BoseFilho, W. W. \& Canto, R. B. - "Processo de prensagem isostática do PTFE em múltiplos passos para extração de ar e redução de defeitos de processamento", in: Anais do $20^{\circ}$ Congresso Brasileiro de Engenharia e Ciência dos Materiais, p.11545, Joinville - SC, nov (2012).

18. Sciuti, V. F.; Angélico, R. A.; Schmitt, N. \& Canto, R. B. "Identification of the heat transfer parameters of the cold pressed PTFE", in: Anais do 22nd International Congress of Mechanical Engineering, p.8, Ribeirão Preto - SP, nov (2013).

19. Riul, C.; Canto, R. B.; Tita, V. \& Carvalho, J. - "Process parameters investigation on the fibre-matrix adhesion of continuous fibres reinforced polytetrafluoroethylene (PTFE)", in: Anais do 17th International Conference on Composite Materials, Edinburgh-UK, july (2009).

20. Riul, C.; Tita, V.; Carvalho, J. \& Canto, R. B. - Compos. Sci. Technol., 72, p.1451 (2012). http://dx.doi.org/10.1016/j. compscitech.2012.05.021.

21. Baker Jr, B. B. \& Kasprzak, D. J. - Polym. Degrad. Stabil., 42, p.181 (1993). http://dx.doi.org/10.1016/01413910(93)90111-U.

22. Sperati, C.A. - "Physical constants of poly (tetrafluorethylene)", in: Polymer Handbook, Brandrup, J.; Immergut, E. H. \& Grulke, E. A. (eds.), Wiley-Interscience Publication, New York (1975).

23. Davidson, T.; Gounder, R. N.; Weber, D. K. \& Wecker, S. M. - "A perspective on solid state microstructure in polytetrafluoroethylene", in: Fluoropolymers 2: Properties, cap.1, Gareth Hougham, Patrick E. Cassidy, Ken Johns and Theodore Davidson (eds), Plenum Press, New York (1999).

Enviado: Fev. 09, 2014

Reenviado: Abr. 23, 2014 Aceito: Abr. 25, 2014 